\title{
Cochlea-Implantation - von der Taubheit in die Welt der Hörenden
}

\author{
Adrian Dalbert ${ }^{a}$, Dorothe Veraguth ${ }^{b}$, Alexander Huber ${ }^{c}$

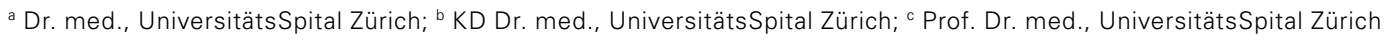

Hochgradige Schwerhörigkeit oder Taubheit ist sowohl bei Kindern als auch bei Erwachsenen eine Erkrankung mit schweren Konsequenzen für die Betroffenen. Mittels Cochlea-Implantation kann ihnen ein Einstieg oder eine Rückkehr in die Welt der Hörenden ermöglicht werden, was neben dem individuellen Nutzen auch von gesellschaftlicher Bedeutung ist.

\section{Schwerhörigkeit und ihre Folgen}

«Blindheit trennt von den Dingen, Taubheit von den Menschen.» Dieser Satz von Helen Keller, einer taubblinden amerikanischen Schriftstellerin, unterstreicht eindrücklich, dass die Taubheit für die betroffenen Kinder und Erwachsenen eine äusserst einschneidende Erkrankung ist. Ungefähr ein bis drei von 1000 Kindern werden taub geboren und ein weiteres von 1000 Kindern ertaubt, bis es das Erwachsenenalter erreicht. Von den über 65-Jährigen leidet ungefähr ein Drittel an einer behandlungsbedürftigen Innenohrschwerhörigkeit - ein Anteil, der mit höherem Alter weiter steigt

Schwerhörigkeiten werden unterteilt in Schallleitungsschwerhörigkeiten, wobei es sich meist um Mittelohrerkrankungen handelt, und Innenohrschwerhörigkeiten. Während bei den Schallleitungsschwerhörigkeiten oft verschiedene Behandlungsmöglichkeiten bestehen (z.B. Paukenröhrchen, Mittelohrprothesen, knochenverankerte Hörgeräte), sind bei leicht- bis mittelgradigen Innenohrschwerhörigkeiten Hörgeräte praktisch die einzige Option. Bei hochgradigen Innenohrschwerhörigkeiten oder Taubheit fällt jedoch auch diese Möglichkeit weg. Die Betroffenen sind trotz Hörgeräten in ihrem Sprachverständnis stark eingeschränkt und häufig auf Lippenlesen sowie Gebärdensprache angewiesen. Eine lautsprachliche Kommunikation ist zunehmend unmöglich. Bei Kindern, die taub geboren sind, bleibt die Sprachentwicklung weitgehend aus. Menschen mit hochgradiger Schwerhörigkeit oder Taubheit nach dem Spracherwerb ziehen sich oft aus der Welt der Hörenden zurück, was häufig neben sozialen Einschränkungen und psychischen Fol- gen auch erheblichen Einfluss auf die schulische oder berufliche Laufbahn hat.

\section{Cochlea-Implantat - die bisher einzige "Sinnesorgan-Prothese»}

Für die Betroffenen ist die Cochlea-Implantation der einzige Weg in die Welt der Hörenden. Das Cochlea-Implantat funktioniert, indem der Hörnerv direkt mittels elektrischer Impulse stimuliert wird. Dies erfolgt über eine Elektrode, die in einer ungefähr 90-minütigen Operation in die Hörschnecke eingelegt wird. Es handelt sich beim Cochlea-Implantat um die bisher einzige Prothese, welche ein Sinnesorgan ersetzen kann.

Die ersten Cochlea-Implantationen erfolgten vor 40 Jahren, ab Beginn der 90er Jahre nahmen sie stetig zu. Im Jahr 1996 erhielten in der Schweiz 42 Patienten Cochlea-Implantate, im Jahr 2016 waren es 243. Die Zahl der Patienten, welche von dieser Behandlung profitieren konnten, hat sich damit in dieser Zeitspanne fast versechsfacht.

Mit dem Cochlea-Implantat erreicht eine grosse Mehrheit der Patienten ein so gutes Sprachverständnis, dass sie am normalen Arbeitsleben teilnehmen können. Bei

Die Zahl der Patienten, welche von einer Cochlea-Implantation profitieren können, hat sich in den letzten 20 Jahren beinahe versechsfacht.

taub geborenen Kindern sollte eine Implantation möglichst innerhalb der ersten beiden Lebensjahre erfolgen, weil so meist eine gute Lautsprachentwicklung und eine Einschulung in eine Regelklasse möglich sind. Damit dies gelingt und schwerhörige respektive taube Kinder möglichst früh entdeckt werden, wurde 
1999 in der Schweiz das flächendeckende Hörscreening eingeführt. Auch dies ist eine Massnahme, welche wie die Cochlea-Implantation zwar Kosten im Gesundheitswesen verursacht, aber neben einem grossen Nutzen für die Betroffenen langfristig auch der Gesellschaft Sonder- und Umschulungen sowie Renten und andere Unterstützungsleistungen einspart. Die folgenden beiden Patientenbeispiele sollen dies veranschaulichen.

\section{Mit Cochlea-Implantaten zur passionierten Reiterin und Bankkauffrau}

T. W. kam im Sommer 1998 nach einer unauffälligen Schwangerschaft zur Welt. In ihrer Familie waren keine Schwerhörigkeiten bekannt. Die Eltern bemerkten jedoch, dass T. W. nur auf visuelle Reize reagierte und selbst laute Geräusche sie vollständig kalt liessen. Es wurden daher weitere Abklärungen veranlasst und im Alter von eineinhalb Jahren eine Hirnstammaudiometrie durchgeführt. Die Diagnose bestätigte den Verdacht der Eltern einer hochgradigen Schwerhörigkeit beidseits.

Da T. W. auch mit den zunächst verwendeten Hörgeräten für eine Sprachentwicklung nicht ausreichend hören konnte, wurde mit den Eltern die Möglichkeit einer Cochlea-Implantation besprochen und bei T. W. im Alter von zwei Jahren durchgeführt.

Inzwischen ist T. W. 19 Jahre alt und Bankkauffrau. Nach der Cochlea-Implantation konnte sie den Rückstand in der Sprachentwicklung aufholen. Sie hat mit Unterstützung durch den audiopädagogischen Dienst die normale Schulausbildung durchlaufen und sowohl die Sekundarschule als auch die Lehre erfolgreich abgeschlossen.

Auch neben Schule und Beruf ist T. W. eine aktive, junge Frau, so dass sie u.a. auch bei ihrem liebsten Hobby, dem Reiten, auf ihre durch die Cochlea-Implantate ermöglichte akustische Wahrnehmung angewiesen ist.

Dr. med. Adrian Dalbert

UniversitätsSpital Zürich

Klinik für Ohren-, Nasen-,

Hals- und Gesichtschirurgie

Frauenklinikstrasse 24

CH-8091 Zürich

Tel. 0442555900

Fax 0442554164

adrian.dalbert[at]usz.ch lich Hörgeräte. Wegen der weiteren Abnahme seines Gehörs war jedoch seine Kommunikationsfähigkeit auch mit Hörgeräten zunehmend eingeschränkt, so dass sich M. P. für eine Cochlea-Implantation, zuerst auf einer Seite, entschied. Aufgrund der deutlichen Verbesserung mit dem Implantat und der weiteren Verschlechterung der Hörleistung am Gegenohr wurde etwas später auch die zweite Seite mit einem CochleaImplantat versorgt.

Mit dem Behandlungsergebnis ist M. P. sehr zufrieden. In seinem sehr anspruchsvollen beruflichen Umfeld als Anästhesie-Kaderarzt mit Schockraum- und Notfalleinsätzen sowie häufiger Tätigkeit im Operationssaal kommt er problemlos zurecht. Dieser berufliche Alltag wäre ohne seine Cochlea-Implantate nicht mehr denkbar.

\section{Zusammenfassung}

Mit der Cochlea-Implantation können Patienten mit hochgradiger Schwerhörigkeit oder Taubheit erfolgreich behandelt werden und Möglichkeiten zur lautsprachlichen Kommunikation geschaffen respektive wiederhergestellt werden. Die Zahl der Patienten, welche von einer Cochlea-Implantation profitieren können, hat sich in den letzten 20 Jahren beinahe versechsfacht. Neben dem Gewinn für die direkt Betroffenen resultiert daraus auch ein gesellschaftlicher Nutzen: Kinder, welche frühzeitig versorgt werden, haben gute Voraussetzungen für die Sprachentwicklung und können später meistens in eine Regelklasse eingeschult werden. Erwachsene können häufig in ihren angestammten Berufen weiterarbeiten. Dies war früher und wäre heute weiterhin ohne die Cochlea-Implantation in den meisten Fällen nicht denkbar. Neben dem grossen Verlust individuellen Potenzials, das vielfach nicht ausgeschöpft werden könnte, würde langfristig auch ein finanzieller Verlust für die Gesellschaft resultieren. Denn dem finanziellen Aufwand von etwa 50000 Schweizer Franken, welchen eine Cochlea-Implantation an Gesundheitskosten verursacht, steht ein Vielfaches an langfristigen Einsparungen gegenüber, da Kosten für Sonder- und Umschulungen sowie Renten und andere Unterstützungsleistungen entfallen. 